\title{
Half metallicity in a zigzag double-walled nanotube nanodot: An ab initio prediction
}

\author{
A.J. Du ${ }^{\text {a,b }}$, Z.H. Zhu ${ }^{c}$, C.H. Sun ${ }^{\mathrm{a}, \mathrm{b}}$, Y. Chen ${ }^{\mathrm{d}}$, G.Q. Lu ${ }^{\mathrm{b}}$, Sean C. Smith ${ }^{\mathrm{a}, \mathrm{b}, *}$ \\ ${ }^{a}$ Centre for Computational Molecular Science, Australian Institute for Bioengineering and Nanotechnology (AIBN), Building 75, The University of Queensland, \\ QLD 4072, Brisbane, Australia \\ ${ }^{\mathrm{b}}$ ARC Centre for Functional Nanomaterials, Australian Institute for Bioengineering and Nanotechnology (AIBN), Building 75, The University of Queensland, QLD 4072, \\ Brisbane, Australia \\ ${ }^{\mathrm{c}}$ Division of Chemical Engineering, School of Engineering, The University of Queensland, QLD 4072, Australia \\ ${ }^{\mathrm{d}}$ Research School of Physical Sciences and Engineering, The Australian National University, ACT 0200, Canberra, Australia
}

\section{A R T I C L E I N F O}

\section{Article history:}

Received 3 November 2008

In final form 12 December 2008

Available online 24 December 2008

\begin{abstract}
A B S T R A C T
$A b$ initio density functional calculations were performed to study finite-length zigzag $(7,0) @(16,0)$ double-walled carbon nanotubes (DWCNTs) with H-termination at the open ends. We find that such a DWCNT nanodot displays a very large magnetic moment at the zigzag edges and the ground state displays symmetric anti-ferromagnetic coupling. When an external electric field is applied along the direction of tube axis, a gap is opened for one spin channel, whereas another spin channel remains metallic, i.e. half metallicity occurs. Our results suggest an important new avenue for the development of CNT-based spintronic materials with enhanced properties.
\end{abstract}

(C) 2008 Elsevier B.V. All rights reserved.

\section{Introduction}

The emerging field of spintronics seeks to exploit electronic spin in addition to electronic charge and is igniting a revolution in computer science, information science and many other areas [1,2]. A key challenge stimulating innovation in this area is how to efficiently manipulate the spin polarization of current. A half metal, i.e. a material which filters the current into a single spin channel without any external operation, fully meets this demand $[3,4]$. In the search for novel materials for spintronics applications, singlewalled carbon nanotubes (SWCNTs) have been proposed as promising candidates due to their peculiar electronic structure and properties [5-7]. Currently, most existing efforts have focused on metal doping of SWCNTs and gate control by connecting CNTs with ferromagnetic leads $[5,8,9]$. Recently, zigzag graphene nanoribbons (GNRs) have been reported to carry a spin current response to an external electric field [10-14], which opens a new pathway for the development of novel carbon-based spintronic devices. Additionally, the finite-length zigzag SWCNTs can be considered to be rolled from GNRs [15]. There have been some pioneering studies that show ferromagnetism and anti-ferromagnetism at their zigzag edges [16-18]. Most recently, finite-length zigzag SWCNTs are found to display half metallicity analogous to that observed in zigzag GNRs [19-21].

\footnotetext{
* Corresponding author. Address: Centre for Computational Molecular Science Australian Institute for Bioengineering and Nanotechnology (AIBN), Building 75, The University of Queensland, QLD 4072, Brisbane, Australia. Fax: +61 73346 3992/ 33654623.

E-mail address: s.smith@uq.edu.au (S.C. Smith).
}

As compared to GNRs and finite-length zigzag SWCNTs, zero dimensional finite-length zigzag DWCNT nanodots should in principle possess large magnetic moments due to many zigzag edges at the open ends and might potentially used as a near-optimal molecule magnet. In this letter, we present $a b$ initio density functional theory (DFT) calculations based on the local spin density approximation (LSDA) [22] to explore the electronic structure and magnetic properties of a finite-length $(7,0) @(16,0)$ DWCNT. We find that the ground state displays symmetric anti-ferromagnetic coupling. The opposite local gating effect [10] of the spin states on the two ends of nanotube is seen to occur in the presence of external electric field along the direction of tube axis. These results suggest a new avenue for the future development of nanotube-based spintronic devices. In the next section, we outline our computational method. Section 3 presents our results for ground state of the $(7,0) @(16,0)$ DWCNT and the influence of external electric fields. The last section is devoted to conclusions.

\section{Computational details}

All the calculations were performed using the plane-wave basis Vienna $a b$ initio simulation package (VASP) $[23,24]$ implementing LSDA [22]. The full-potential projector augmented wave method (PAW) $[25,26]$ is used to describe the electronic-ion-core interaction. The finite-length $(7,0) @(16,0)$ DWCNT was saturated with $\mathrm{H}$ atoms at all open ends, containing totally 322 atoms. The super cell $(23 \AA \times 23 \AA \times 22.5 \AA)$ is large enough to ensure the separation of interaction between periodic images is negligible. Gamma point only is used for the sampling of one-dimensional Brillouin zone. The cutoff energies for plane waves are chosen to be $500 \mathrm{eV}$ and 
the convergence tolerance for the force on each atom is $0.01 \mathrm{eV} / \AA$ during geometry optimization.

\section{Results and discussion}

A finite-length $(7,0) @(16,0)$ DWCNT with six carbon atoms along the tube axis was built and both open ends were saturated with hydrogen atoms. The structures were first fully optimized using non spin-polarized DFT calculations as shown in Fig. 1a and $b$ (the nonmagnetic (NM) phase). The finite-length $(7,0) @(16,0)$ DWCNT remained largely undeformed and the C-C and $\mathrm{C}-\mathrm{H}$ bond length at open ends are $1.40 \AA$ and $1.10 \AA$, respectively. As compared to infinite $(7,0) @(16,0)$ DWCNT, the C-C bond length at open end is around $0.1-0.2 \AA$ shorter than that in middle part. To verify the stability of finite-length $(7,0) @(16,0)$ DWCNT, we performed an empirical molecular dynamic simulation at room temperature based the Brenner $\mathrm{C}-\mathrm{H}$ potential [27]. It was found that the finite-length $(7,0) @(16,0)$ DWCNT is quite stable. The relaxed equilibrium DWCNT structure was then used as the initial configuration for further optimizing ferromagnetic (FM) and antiferromagnetic (AFM) states. Additionally, AFM phases with different and same spin orientation for inner and outer tube (we called anti-symmetric and symmetric AFM-1 and AFM-2 phases) were considered as shown in Fig. $2 b$ and c. The energy differences between FM, AFM-1 and AFM-2 and NM were calculated to be $-673,-731$ and $-746 \mathrm{meV}$, respectively. The total magnetic moment of FM phase for finite-length $(7,0) @(16,0)$ DWCNT is as high as $9 \mu \mathrm{B}$, which allows this DWCNT nanodot to be regarded as a molecular magnet. Additionally, the total energy of AFM-1 is around $60 \mathrm{meV}$ lower than FM phase. The energy difference be- a

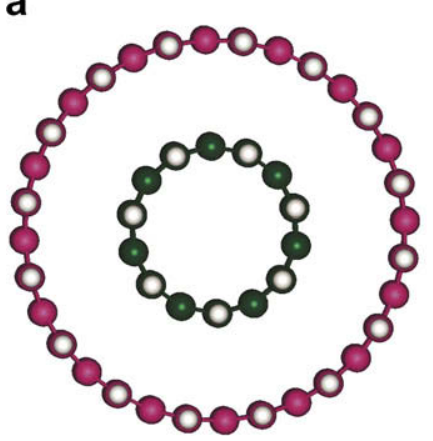

b

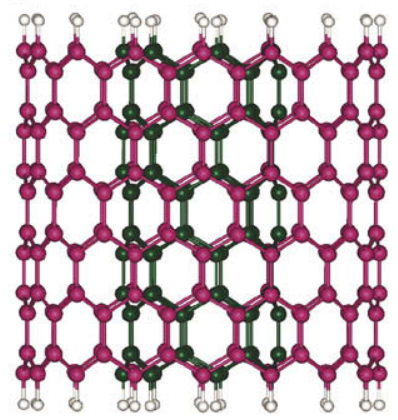

Fig. 1. Optimized geometry for a $(7,0) @(16,0)$ DWCNT. (a) Top view and (b) side view. tween AFM-1 and AFM-2 is $15 \mathrm{meV}$, which indicate symmetric AFM-2 phase is the ground state of finite-length open ended $(7,0) @(16,0)$ DWCNT. One may doubt the effects of using a truncated plane-wave basis for describing a finite-sized molecular system. However, high level full-electron methods are not feasible for such a large system containing totally 322 atoms.

In Fig. 2a-c, we present three dimensional iso-surfaces (the isovalue is $\left.0.01 \mathrm{e} / \AA^{3}\right)$ of spin charge density magnetization $\left(\rho_{\uparrow}-\rho_{\downarrow}\right)$ in a finite-length $(7,0) @(16,0)$ DWCNT for the FM, asymmetric and symmetric AFM phase (AFM-1 and AFM-2), respectively. Clearly, the magnetic charge density is mainly localized at the zigzag edge of two open ends in the FM phase. In the AFM phase, spin-up and spin-down charge density was distributed at each open end and decay from the edges to the middle. The local magnetic moment was calculated to be around $0.15 \mu \mathrm{B}$ per edge carbon atom and the value in the middle is nearly zero. This indicated that the magnetic moment mainly arise from the zigzag edge.

Having explored the electronic properties for a finite-length $(7,0) @(16,0)$ zigzag DWCNT, we now turn to study the effect of external electric field along tube axis. In our study, an artificial dipole sheet is introduced in the middle of the vacuum part in the periodic supercell to simulate electric field [28]. The strength of field $E$ is chosen to be varied from 0.0 to $0.60 \mathrm{~V} / \AA$, which are weak and strong enough to avoid artificial field emission and make significant change of the electronic structure, respectively. Fig. 3 presents plots of spin-up and spin-down density of states in the symmetric anti-ferromagnetic ground state (AFM-2) for a finitelength $(7,0) @(16,0)$ DWCNT at different external electric fields. Clearly, it shows zero Homo-Lumo gap in the discrete energy-level of DWCNT nanodot (i.e. metallic behavior) in the absence of external electric field. We should note both single-walled $(7,0)$ and $(16,0)$ carbon nanotubes are semi-conducting. The metallicity in DWCNT is mainly due to the effect of curvature difference and interwall spacing as reported in an earlier study for infinite length DWCNTs [29]. Here finite-length open ended $(7,0) @(16,0)$ DWCNT show similar behavior as in the case of infinite zigzag nanotube. With the application of the external field of $0.1 \mathrm{~V} / \AA$ along the direction of tube axis, spin-up channel remained unchanged but the density of state around Fermi level for spin-down channel was slightly decreased. As the field strength is increase up to $0.3-0.5 \mathrm{~V} / \mathrm{A}$, spin-down channel open a gap and become semiconducting, whereas spin-up channel remains metallic, i.e. complete spin-polarization around the Fermi level is evidenced. As shown in Fig. 2c, each spin-up and spin-down density is mainly localized to one end of the tube. With the introduction of electric filed, the spin density at two ends is reduced significantly and the systems finally becomes diamagnetic at high electric filed, a feature that has also been observed in GNRs [30,31]. This can be a

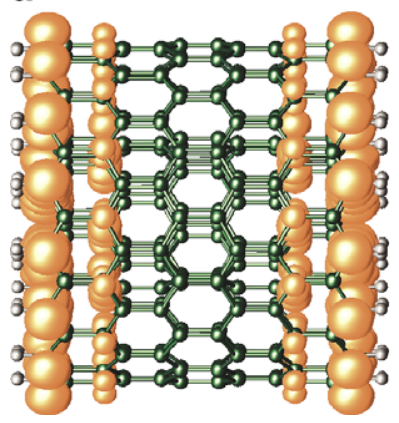

b

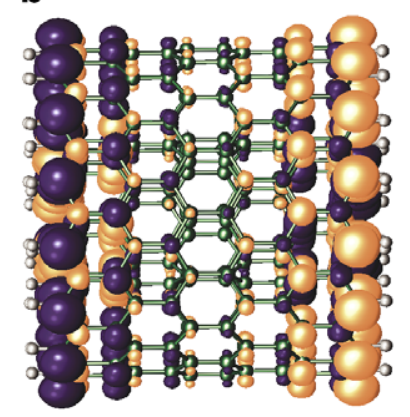

C

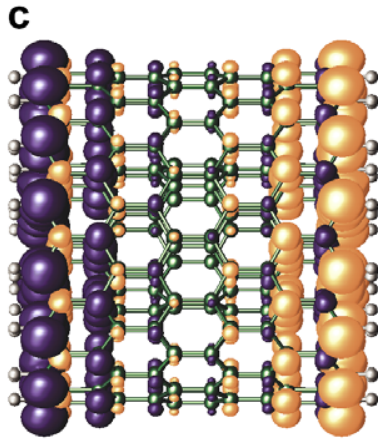

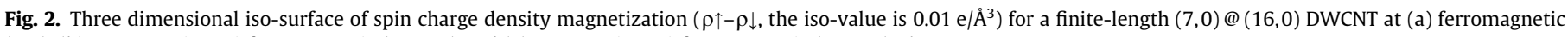
(FM), (b) asymmetric anti-ferromagnetic (AFM-1) and (c) symmetric anti-ferromagnetic (AFM-2) phases. 
a

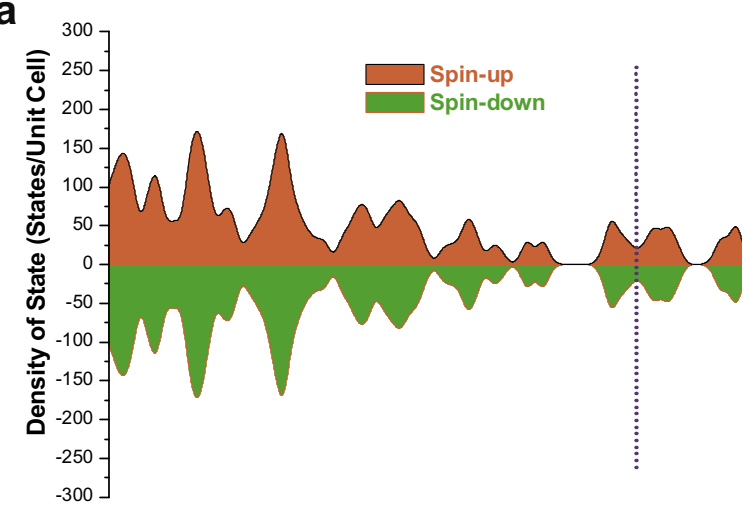

C

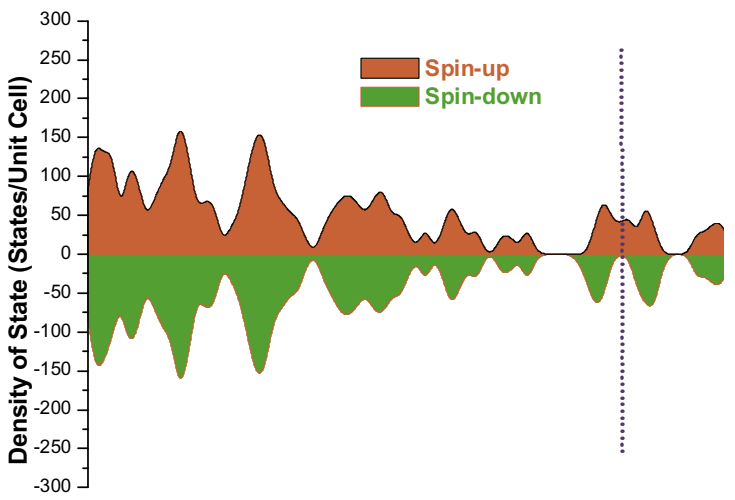

b

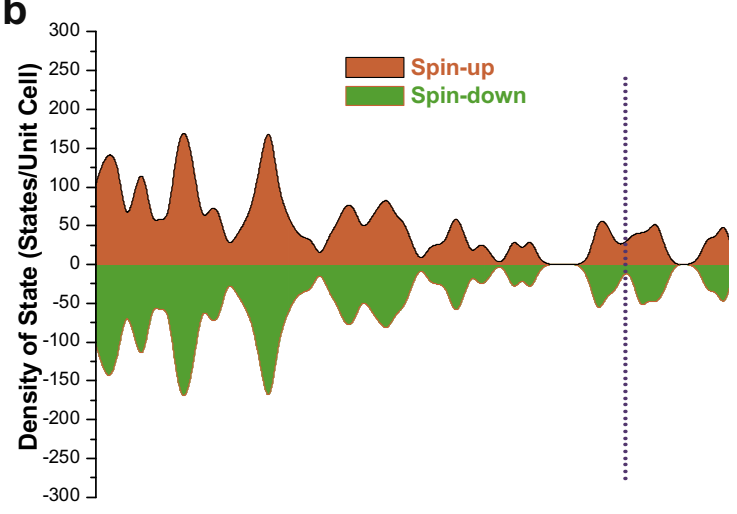

d

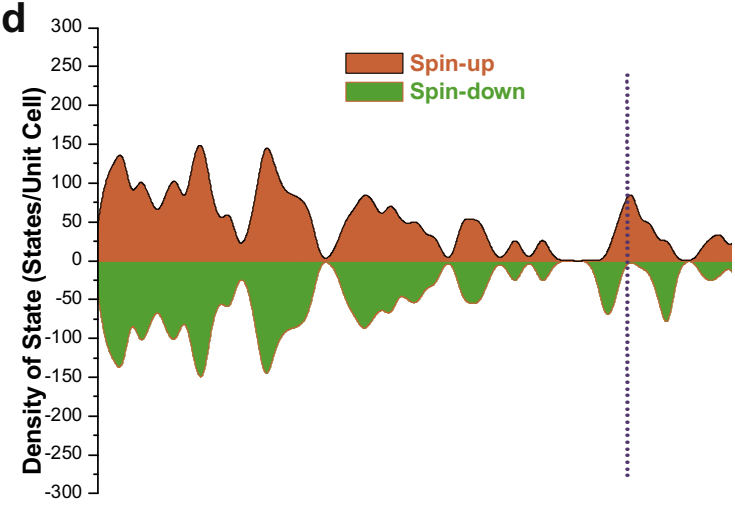

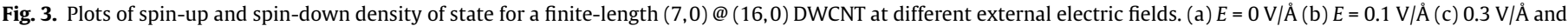
(d) $E=0.5 \mathrm{~V} / \AA$.

understood by the fact that increasing the external field will result in energy-level shifts of opposite signs for the separated spin-ordered edge states and the energy gap opening for one spin channel. Apparently, such half metallicity in finite-length $(7,0) @(16,0)$ DWCNT will lead to spin transport anisotropy and suggests potential applications for finite-length zigzag DWCNT nanodots in spintronic devices.

\section{Conclusions}

In summary, we have implemented $a b$ initio DFT calculations to demonstrate that the ground state of a finite-length $(7,0) @(16,0)$ DWCNT displays symmetric anti-ferromagnetic coupling and exhibits half metallicity under the influence of an external electric field along the tube axis. These results highlight an intriguing new avenue for future investigations in the development of nanotubebased nanospintronic devices.

\section{Acknowledgements}

We acknowledge generous grants of high-performance computer time from both the Computational Molecular Science cluster computing facility at The University of Queensland and the Australian Partnership for Advanced Computing (APAC) National Facility. The authors also greatly appreciate the financial support by Australian Research Council through the ARC Centre for Functional Nanomaterials.

\section{References}

1] S.A. Wolf et al., Science 294 (2001) 1488.

[2] D.D. Awschalom, M.E. Flatt, Nature Phys. 3 (2007) 153.

[3] C. Felser, G.H. Fecher, B. Balke, Angew. Chem., Int. Ed. 46 (2007) 668

[4] E. Durgun, D. Cakir, N. Akman, S. Ciraci, Phys. Rev. Lett. 99 (2007) 256806.

[5] A. Cottet et al., Semicond. Sci. Technol. 21 (2006) S78.

[6] A. Brataas, Nature 452 (2008) 419.

[7] F. Kuemmeth, S. IIani, D.C. Ralph, P.L. McEuen, Nature 452 (2008) 448

[8] C.K. Yang, J.J. Zhao, J.P. Lu, Nano Lett. 4 (2004) 561.

[9] C.K. Yang, J.J. Zhao, J.P. Lu, Phys. Rev. Lett. 90 (2003) 257203.

[10] Y.W. Son, M.L. Cohen, S.G. Louie, Nature 444 (2006) 347.

[11] Y.W. Son, M.L. Cohen, S.G. Louie, Phys. Rev. Lett. 97 (2006) 216803

[12] S. Dutta, S.K. Pati, J. Phys. Chem. B 112 (2008) 1333.

[13] O. Hod, V. Barone, G.E. Scuseria, Phys. Rev. B 77 (2008) 035411.

[14] D.E. Jiang, B.G. Sumpter, S. Dai, J. Chem. Phys. 127 (2007) 124703.

[15] A.J. Du, S.C. Smith, G.Q. Lu, Nano Lett. 7 (2007) 3349.

[16] K. Kusakabe, M. Maruyama, Phys. Rev. B 67 (2003) 092406.

[17] S. Okada, A. Oshiyama, J. Phys. Soc. Jpn. 72 (2003) 1510.

[18] Z.F. Chen, D.E. Jiang, X. Lu, H.F. Bettinger, S. Dai, P.V. Schleyer, K.N. Houk, Org. Lett. 9 (2007) 5449.

[19] A.J. Du, Y. Chen, G.Q. Lu, S.C. Smith, Appl. Phys. Lett. 93 (2008) 073101.

[20] A. Mananes, F. Duque, A. Ayuela, M.J. Lopez, J.A. Alonso, Phys. Rev. B 78 (2008) 035432.

[21] O. Hod, G.E. Scuseria, ACS Nano 2 (2008) 2243.

[22] D.M. Ceperley, B.J. Alder, Phys. Rev. Lett. 45 (1980) 566

[23] G. Kresse, J. Furthmuller, Comput. Mater. Sci. 6 (1996) 15

[24] G. Kresse, J. Furthmuller, Phys. Rev. B 54 (1996) 11169.

[25] P.E. Blochl, Phys. Rev. B 50 (1994) 17953.

[26] G. Kresse, D. Joubert, Phys. Rev. B 9 (1999) 1758.

[27] D.W. Brenner, O.A. Shenderova, J.A. Harrison, S.J. Stuart, B. Ni, S.B. Sinnott, J. Phys. Condens. Matter 14 (2002) 783.

[28] J. Neugebauer, M. Scheffler, Phys. Rev. B 46 (1992) 16067.

[29] S. Okada, A. Oshiyama, Phys. Rev. Lett. 91 (2003) 216801.

[30] E. Rudberg, P. Salek, Y. Luo, Nano Lett. 7 (2007) 2211.

[31] E.J. Kan, Z.Y. Li, J.L. Yang, JG. Hou, Appl. Phys. Lett. 91 (2007) 243116. 